\title{
PDDA-Montmorillonite Composites Loaded with Ru Nanoparticles: Synthesis, Characterization, and Catalytic Properties in Hydrogenation of 2-Butanone
}

\author{
Ewa M. Serwicka ${ }^{1, *(\mathbb{D})}$, Małgorzata Zimowska ${ }^{1}$ (), Dorota Duraczyńska ${ }^{1}$, \\ Bogna D. Napruszewska ${ }^{1}$, Małgorzata Nattich-Rak ${ }^{1}$, Grzegorz Mordarski ${ }^{1}$, \\ Lidia Lityńska-Dobrzyńska ${ }^{2}$ and Helena Palkova ${ }^{3}$ \\ 1 Jerzy Haber Institute of Catalysis and Surface Chemistry, Polish Academy of Sciences, Niezapominajek 8, \\ 30-239 Krakow, Poland; nczimows@cyf-kr.edu.pl (M.Z.); ncduracz@cyf-kr.edu.pl (D.D.); \\ ncnaprus@cyf-kr.edu.pl (B.D.N.); ncnattic@cyf-kr.edu.pl (M.N.-R.); ncmordar@cyf-kr.edu.pl (G.M.) \\ 2 Institute of Metallurgy and Materials Science, Polish Academy of Sciences, Reymonta 25, \\ 30-059 Krakow, Poland; 1.litynska@imim.pl \\ 3 Institute of Inorganic Chemistry, Slovak Academy of Sciences, Dúbravská cesta 9, \\ SK-845 36 Bratislava, Slovakia; helena.palkova@savba.sk \\ * Correspondence: ncserwic@cyf-kr.edu.pl; Tel.: +48-12-6395-118
}

Received: 11 July 2018; Accepted: 1 August 2018; Published: 4 August 2018

\begin{abstract}
The effect of synthesis parameters on the physicochemical properties of clay/ polydiallyldimethylammonium (PDDA)/Ru composites and their applicability in hydrogenation of 2-butanone under very mild conditions (room temperature, atmospheric pressure, and aqueous solution) was studied. Three synthetic procedures were employed, differing in the order of addition of components and the stage at which metallic Ru species were generated. The materials were characterized with XRD (X-ray diffraction), XRF (X-ray fluorescence), EDS (energy-dispersive spectroscopy), AFM (atomic force microscopy), TEM/HRTEM (transmission electron microscopy/high resolution transmission electron microscopy), and TG/DSC (thermal gravimetry/differential scanning microscopy techniques. The study revealed that the method of composite preparation affects its structural and thermal properties, and controls the distribution and size of Ru particles. All catalysts are active in hydrogenation of 2-butanone. For best catalytic performance ( $100 \%$ conversion within $30 \mathrm{~min}$ ) both the size of Ru particles and the load of polymer had to be optimized. Superior catalytic properties were obtained over the composite with intermediate crystal size and intermediate PDDA load, prepared by generation of metallic Ru species in the polymer solution prior to intercalation. This method offers an easy way of controlling the crystal size by modification of $\mathrm{Ru} / \mathrm{PDDA}$ ratio.
\end{abstract}

Keywords: polymer-clay composite; montmorillonite; polydiallyldimethylammonium; Ru nanoparticles; Ru catalyst; 2-butanone hydrogenation

\section{Introduction}

The use of nanoparticles in the design of catalysts based on noble metals is particularly attractive, because the large surface to volume ratio characteristic of very fine metal species enables efficient utilization of costly active phase [1]. In addition, tailoring of metal nanoparticle size offers means for control of catalytic activity [2,3]. Unfortunately, naked nanoparticles are prone to agglomeration, which hinders their effective use [3,4]. This obstacle may be overcome by deposition of metal nanoparticles on an appropriate support, and/or by stabilizing their dispersion with use of various capping agents [3-7]. To this end, the use of polymers containing functional groups that provide attractive interaction with nanoparticles is of particular interest [8]. In such systems the polymer component affords, 
on one hand, the stabilizing steric bulk framework, and on the other, offers many binding sites capable of simultaneous interaction with nanoparticles. The latter makes nanoparticle immobilization very efficient, even if the interaction with an individual functional group is only weak. The use of polymers as stabilizing agents is of particular advantage in the design of advanced nanomaterials based on clay-related layered silicates, because the latter readily form hybrid structures with polymeric components $[9,10]$. Clay-polymer composites are well recognized for their industrial applications, ranging from heat resistant automotive components to packaging, coating and adhesive materials, aerospace, optical, electronic, and medical devices. Clay minerals, which belong to the family of layered silicates, are especially attractive for the design of composite materials due to their large abundance in nature and unique structural features. The silicate layers, which are the fundamental units of clay minerals, may be easily used as ready-made building blocks in advanced nanocomposite design [11-14]. In particular, synthesis of ternary systems involving clay, polymer and various metal nanoparticles (e.g., Ag, Au, $\mathrm{Rh}, \mathrm{Pd}$, and Fe), as potential catalysts have been reported [15-20].

In the present work we focused our attention on the use of polydiallyldimethylammonium (PDDA) chloride as a polymer stabilizing the catalytically active ruthenium nanoparticles embedded in montmorillonite clay. Montmorillonite is a naturally occurring phyllosilicate mineral, whose lattice is composed of stacked layers built of one octahedral Al-based sheet sandwiched between two Si-based tetrahedral sheets. Isomorphic substitution within the octahedral layer (mainly of $\mathrm{Al}^{3+}$ by $\mathrm{Mg}^{2+}$ ) generates a negative charge, which is compensated by the presence of hydrated cations in the interlayer [21]. These cations undergo facile exchange, which is the basis for rich intercalation chemistry of montmorillonite. PDDA is a cationic polyelectrolyte, used as a coagulant in water purification, as an additive controlling disturbing substances in the paper production, or as a model compound in different areas of polyelectrolyte research [22]. It is particularly suitable for formation of composites with montmorillonite, because the PDDA polycations readily enter the mineral structure via exchange with the charge compensating cations $[15,16]$. The binding of the composite components occurs through electrostatic interactions between the negatively charged sites of clay mineral layers and positively charged groups of the polymer. Ruthenium nanoparticles, the third component of the investigated composites, are known for their catalytic properties, especially in hydrogenation reactions [23]. The aim of this study was to investigate the effect of synthesis parameters on the physicochemical properties of clay/PDDA/Ru composites and their applicability in hydrogenation of 2-butanone under very mild conditions (room temperature, atmospheric pressure, and aqueous solution). Hydrogenation of 2-butanone is a very useful test reaction, because under the adopted conditions it is $100 \%$ selective to 2-butanol, and enables straightforward comparison of catalysts activities without the necessity of addressing the selectivity issue [24].

\section{Materials and Methods}

\subsection{Materials}

The starting montmorillonite used for the preparation of composites, denoted Mt, was the sodium form of the less than $2 \mu \mathrm{m}$ particle size fraction separated by sedimentation from Jelšový Potok (Envigeo Inc., Slovakia, Czech Republic) bentonite, with cation exchange capacity (CEC) of $85 \mathrm{meq} / 100 \mathrm{~g}$. $20.0 \mathrm{wt} \%$ aqueous solution of low molecular weight polydiallyldimethylammonium (PDDA) chloride (Sigma-Aldrich, Poznań, Poland), average $M_{W}=100,000-200,000$, was used after dilution to $0.05 \mathrm{wt} \%$ concentration. $\mathrm{RuCl}_{3} \cdot x \mathrm{H}_{2} \mathrm{O}$ (Sigma-Aldrich) was used as ruthenium source.

The first step in the synthesis of Mt/PDDA/Ru composites involved preparation of Mt suspension in distilled water $(0.5 \mathrm{~g}$ clay $/ 100 \mathrm{~mL}$ water). The suspension was stirred for $24 \mathrm{~h}$ at room temperature to enhance Mt exfoliation. Next, $0.2 \mathrm{wt} \% \mathrm{RuCl}_{3}$ aqueous solution in the amount corresponding to $2 \mathrm{wt} \%$ of $\mathrm{Ru}$ with respect to Mt component, was mixed with different amounts of $0.05 \mathrm{wt} \%$ PDDA aqueous solution for $1 \mathrm{~h}$ and reduced with excess $\mathrm{NaBH}_{4}$ methanolic solution (molar ratio $\mathrm{NaBH}_{4} / \mathrm{Ru}=100$ ). The mixture was added drop-wise to the Mt suspension at room temperature and left under stirring 
for $24 \mathrm{~h}$. Then the product was retrieved and washed free of $\mathrm{Cl}^{-}$by centrifugation till negative reaction of the supernatant with $\mathrm{AgNO}_{3}$, and stored as aqueous gel. This procedure is referred to as method $\mathrm{I}$. Samples prepared this way, are denoted Mt/(Ru + PDDA_x ), where $x$ is the PDDA/Mt weight ratio, equal $0.01,0.025,0.05,0.1$, or 0.25 . Theoretically, the complete cation exchange requires the PDDA $/ \mathrm{Mt}$ weight ratio of 0.14 . For the sake of comparison, additional samples with PDDA/Mt ratio of 0.05 and 0.25 were obtained, by changing the sequence of synthetic steps. Thus, in method II, the preparative route followed the path of method I, except that the reduction with $\mathrm{NaBH}_{4}$ was carried out in a post-synthesis manner, in the final stage of the composite manufacturing. The resulting two samples are referred to as $\mathrm{Mt} /\left(\mathrm{Ru}+\mathrm{PDDA} \_x\right)$ post. In method III the Mt component was first intercalated with polycations by mixing with the PDDA solution and stirred for $24 \mathrm{~h}$. Subsequently, the resulting Mt/PDDA_ $x$ complex was mixed with $\mathrm{RuCl}_{3} \cdot x \mathrm{H}_{2} \mathrm{O}$ aqueous solution $(2 \mathrm{wt} \%$ of Ru with respect to $\mathrm{Mt}$ ) for $24 \mathrm{~h}$ and the whole mixture subjected to reduction with $\mathrm{NaBH}_{4}$. The samples obtained this way are referred to as Mt/PDDA $\_x / R u$. The main steps of each procedure are illustrated in Figure 1 .

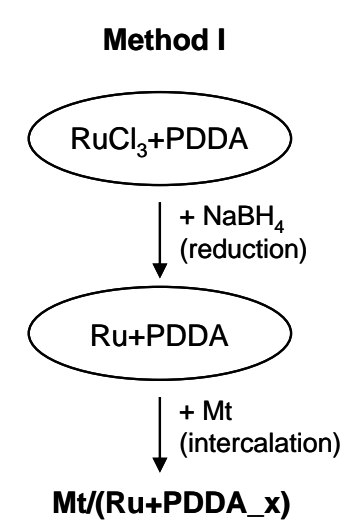

$\mathrm{x}=\mathrm{PDDA} / \mathrm{Mt}$ weight ratio

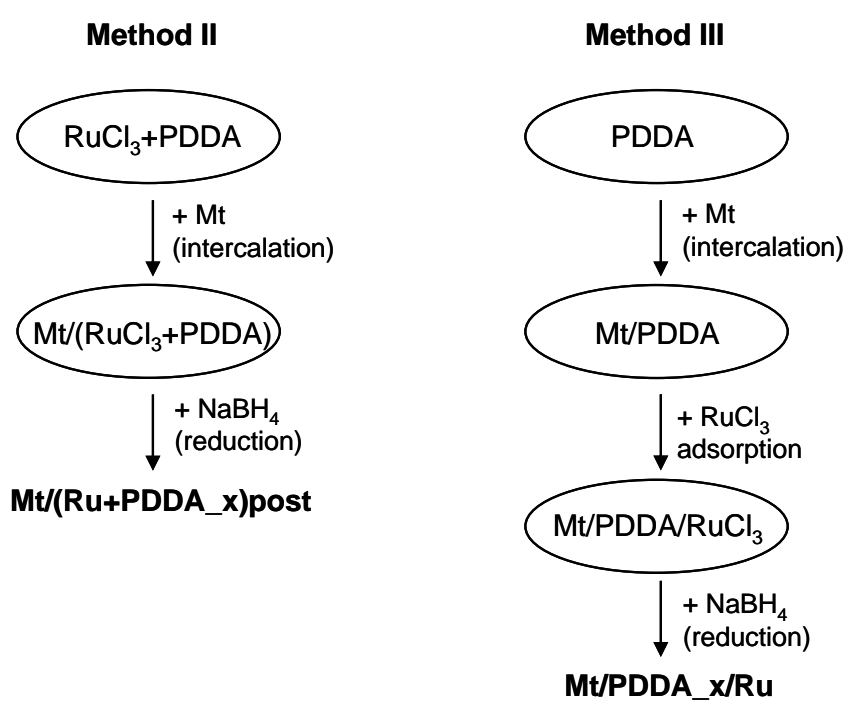

Mt/PDDA_x/Ru

Figure 1. Schematic illustration of catalysts synthesis methods.

\subsection{Methods}

X-ray diffraction (XRD) patterns were recorded using X'Pert PRO MPD diffractometer (PANalytical, Almelo, the Netherlands) with $\mathrm{CuK} \alpha$ radiation $(40 \mathrm{kV}, 30 \mathrm{~mA})$ selected by a nickel monochromator in the diffraction beam, with a step size $0.05^{\circ}$. The samples were prepared as oriented thin films by drying a suspension of clay composite on a glass slide.

$\mathrm{X}$-ray fluorescence (XRF) analysis, used for the determination of Ru content, was carried out with Skyray EDX $3600 \mathrm{H}$ spectrometer (Skyray, Braintree, MA, USA), equipped with X-ray source (40 kV, $220 \mu \mathrm{A}$ ). The $\mathrm{Na} / \mathrm{Si}$ ratio in the investigated samples was determined with aid of a JSM-7500F Field Emission scanning electron microscope (JEOL, Akishima, Japan) equipped with an energy-dispersive (EDS) analyzer (Link ISIS, Oxford Instruments, London, UK).

Atomic force microscopy (AFM) measurements of Ru nanoparticles adsorption on mica surface were carried out using the NT-MDT IX71 device with the SMENA scanning head (NT-MDT, Russia). The measurements were performed in semi-contact mode using silicon probes. Ru suspension in PDDA aqueous solution was allowed to adsorb on mica surface for $60 \mathrm{~min}$ and then the substrate was removed and rinsed for $30 \mathrm{~s}$ with distilled water.

Transmission and high resolution transmission electron microscopic studies (TEM and HRTEM, respectively) were performed with FEI Tecnai G2 (FEI, Eindhoven, the Netherlands) transmission electron microscope. The dominant size of Ru particles in the sample was estimated from the assessment of at least 250 particles in selected areas of enlarged TEM micrographs. 
Thermal analysis was carried out with a DSC/TG Netzsch STA 409 PC LUXX apparatus (Netzch, Germany), in the temperature range $30-1000{ }^{\circ} \mathrm{C}$, at a heating rate of $10{ }^{\circ} \mathrm{C} / \mathrm{min}$ and in the flow of air $(40 \mathrm{~mL} / \mathrm{min})$. Theoretical weight losses were calculated under the assumption that the mass loss in the $200-1000{ }^{\circ} \mathrm{C}$ range is due to the elimination of the organic component (PDDA) and to the dehydroxylation of Mt layers. The quantitative contribution of the latter was calculated from the TG curve of Mt sample. PDDA is a polymer of $100 \%$ cationicity, therefore for the calculation of the initial mass of PDDA-exchanged samples it was assumed that the exchange involves replacement of one $\mathrm{Na}^{+}$ with one $\mathrm{PDDA}^{+}$monomer.

2-Butanone hydrogenation experiments were carried out in an agitated batch glass reactor (PARR 5100, Parr Instrument Company, Moline, IL, USA) at constant pressure of hydrogen (1 bar) and room temperature. In a typical hydrogenation test the catalyst, containing $4 \times 10^{-6}$ mole of $\mathrm{Ru}$, was added to the solution of $3 \times 10^{-3}$ mole of 2-butanone in $50 \mathrm{~mL}$ water. Prior to the catalytic test the system was purged with nitrogen. Subsequently hydrogen was introduced and the reagents vigorously stirred (800 rpm) for $0.5 \mathrm{~h}$. A gas chromatograph (Clarus 500, Perkin Elmer, Boston, MA, USA) equipped with CP-Chirasil-Dex CB column (Varian Inc., Walnut Creek, CA, USA) was used for analyzing the composition of reaction mixture. Catalytic results were not affected by an increase of the agitation speed, which pointed to the absence of diffusion limitations.

\section{Results and Discussion}

\subsection{Physicochemical Characterization}

Prior to studying the Ru-containing composites, the effect of PDDA intercalation on the XRD pattern of the parent Mt was examined (Figure 2a).

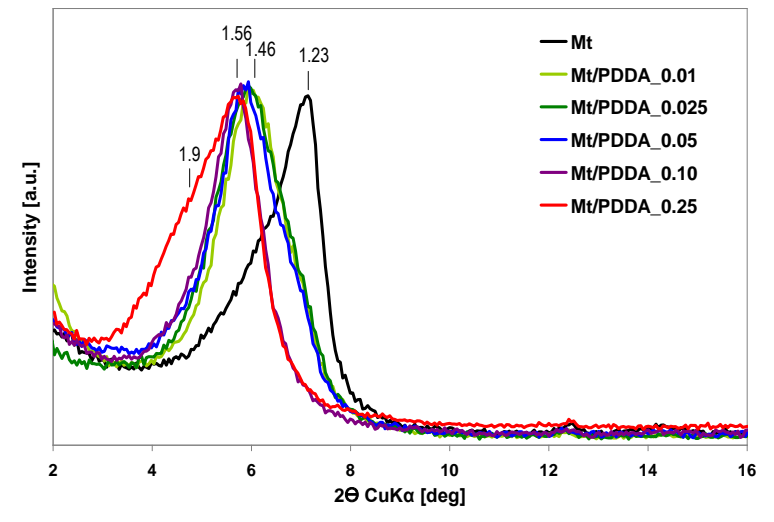

(a)

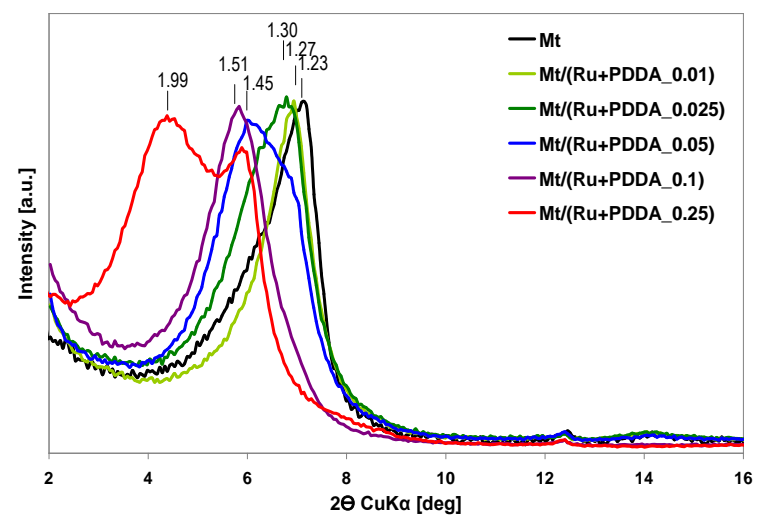

(b)

Figure 2. (a) Effect of polydiallyldimethylammonium (PDDA) loading on the XRD pattern of parent montmorillonite; (b) Effect of Ru insertion by method I on XRD pattern of montmorillonite (Mt) intercalated with PDDA cations (PDDA loadings as in Figure 2a).

The basal spacings of PDDA-intercalated samples, reported in Table 1, show a shift from $d_{001}=1.23 \mathrm{~nm}$, characteristic of the sodium form of montmorillonite, to $1.46-1.56 \mathrm{~nm}$, typical of PDDA-clay complex in which a single layer of polymer chains, adhering to the basal planes of montmorillonite, is formed [25-27]. The effect is associated with the 100\% cationicity of PDDA, which causes strong attraction with clay layers and favors extended polymer conformation, with few loops and trains [26]. Noteworthy, in samples with the lowest PDDA loadings, the basal reflection displays an asymmetry in the direction of higher $2 \Theta$ values, towards the basal reflection of the parent clay mineral, indicating the presence of not completely exchanged interlayers. Indeed, the chemical analysis shows that these samples retain a certain amount of sodium. This is not surprising, since in most 
samples the amount of PDDA used for intercalation was less than the CEC (Table 1). However, the data in Table 1 show that the Na content, especially in samples with less PDDA, is much lower than that expected to be left in the materials after complete exchange with PDDA cations (the amount of PDDA used in the synthesis corresponded to ca. 7\%, 18\%, and 36\% of CEC for Mt/(Ru + PDDA_0.01), Mt/(Ru + PDDA_0.025), and Mt/(Ru + PDDA_0.05), respectively. Presumably, in view of the acidic $\mathrm{pH}$ of PDDACl solution $(\mathrm{pH}=2)$, a part of sodium becomes exchanged with hydronium ions. Under the adopted conditions the interlayer spacing of $\mathrm{H}$-form of montmorillonite is ca. $1.5 \mathrm{~nm}$, therefore we propose that the basal reflection corresponding to this value is, especially in samples with low PDDA loadings, partly due to the presence of $\mathrm{H}_{3} \mathrm{O}^{+}$in the Mt interlayer. In the Mt/PDDA_0.25 sample, loaded with the highest PDDA content employed in this work a shoulder at lower $2 \Theta$ appears, corresponding to ca. $1.9 \mathrm{~nm}$ interlayer distance, suggesting that a part of intercalated polymer forms a double layer $[26,27]$.

Table 1. Basal spacing, $\mathrm{Na} / \mathrm{Si}$ atomic ratio, $\mathrm{Ru}$ content, mass loss in the $200-1000{ }^{\circ} \mathrm{C}$ range, and 2-butanone conversion of studied samples.

\begin{tabular}{|c|c|c|c|c|c|}
\hline Sample & $\mathrm{d}_{001}(\mathrm{~nm})$ & $\mathrm{Na} / \mathrm{Si}$ & Ru (wt \%) & $\Delta \mathrm{TG}_{200-1000}(\mathrm{wt} \%)$ & 2-Butanone Conversion ( $\%)$ \\
\hline Mt & 1.25 & 0.092 & - & 5.6 & 0 \\
\hline Mt/PDDA_0.01 & 1.46 & 0.021 & - & $7.3(6.3)^{1}$ & n.d ${ }^{2}$ \\
\hline Mt/PDDA_0.05 & 1.50 & 0.009 & - & $9.7(9.2)$ & 0 \\
\hline Mt/PDDA_0.1 & 1.56 & 0.003 & - & $12.3(12.5)$ & n.d. \\
\hline Mt/PDDA_0.25 & 1.53 & 0.001 & - & $15.9(21.3,15.0 *)$ & n.d. \\
\hline $\mathrm{Mt} /(\mathrm{Ru}+$ PDDA_0.05) & $1.45(1.30)$ & 0.044 & 1.95 & $8.2(9.0)$ & 100 \\
\hline $\mathrm{Mt} /(\mathrm{Ru}+$ PDDA_0.1) & 1.51 & 0.028 & 1.81 & $12.0(12.4)$ & 45 \\
\hline $\mathrm{Mt} /(\mathrm{Ru}+$ PDDA_0.25) & $1.99(1.50)$ & 0.008 & 1.67 & $19.8\left(21.3,14.7^{*}\right)$ & 13 \\
\hline $\mathrm{Mt} /(\mathrm{Ru}+$ PDDA_0.05)post & 1.49 & 0.046 & 1.74 & $9.6(9.0)$ & 36 \\
\hline $\mathrm{Mt} /(\mathrm{Ru}+$ PDDA_0.25)post & $1.97(1.56)$ & 0.002 & 1.61 & $18.9\left(21.3,14.7^{*}\right)$ & 7 \\
\hline $\mathrm{Mt} /$ PDDA_0.05/Ru & 1.51 & 0.048 & 1.99 & $9.4(9.0)$ & 26 \\
\hline
\end{tabular}

${ }^{1}$ in brackets weight loss expected if all added polymer entered the composite, ${ }^{2}$ n.d.- - not determined; * weight loss expected if the amount of retained polymer corresponds to the CEC of clay.

In the case of method I, in which the reduced Ru species were formed in PDDA prior to intercalation, dispersion of metal particles in the polymer solution has been examined with aid of atomic force microscopy.

Figure 3 shows the representative AFM images recorded for three PDDA loadings: the minimum, the intermediate and the maximum one $(x=0.01,0.05$, and 0.25$)$. It may be seen that with the increase of the relative content of PDDA, Ru nanoparticles become better dispersed. The size of the smallest particles visible in the AFM images is about $20 \mathrm{~nm}$. Such species dominate in the intercalating agent used for Mt/(Ru + PDDA_0.25) preparation, but upon lowering of the relative PDDA content the particles tend to form larger agglomerates, of ca. $60-80 \mathrm{~nm}$ dimension, and such clusters prevail in the suspension used for Mt/(Ru + PDDA_0.01) synthesis. The complex nature of AFM-visible particles is confirmed by the TEM study, described further. 


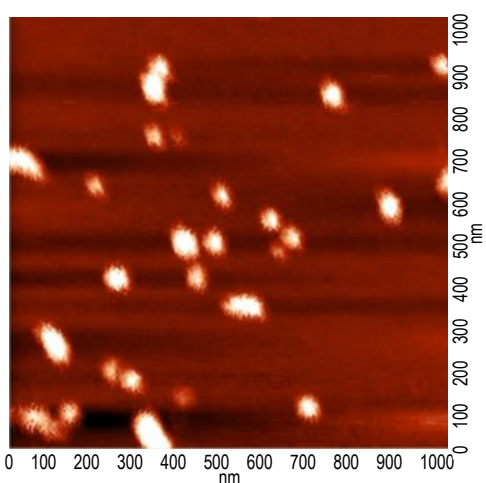

(a)

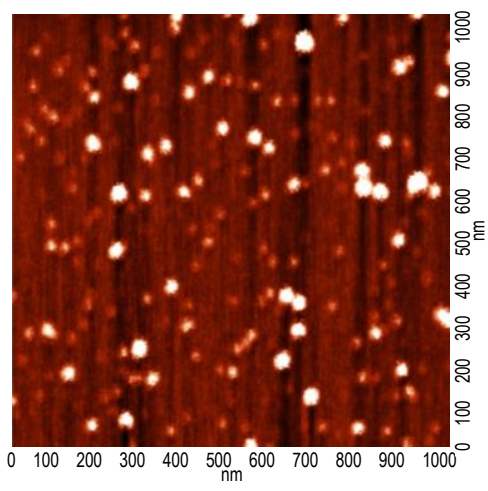

(b)

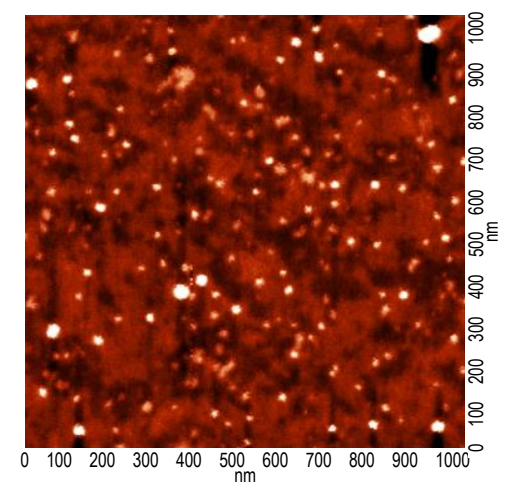

(c)

Figure 3. AFM topography images of Ru suspensions in PDDA solutions used for intercalation of (a) $\mathrm{Mt} /(\mathrm{Ru}+$ PDDA_0.01); (b) Mt/(Ru + PDDA_0.05); and (c) Mt/(Ru + PDDA_0.25) samples.

The influence of Ru addition by method I on the structural features of PDDA/clay intercalates is illustrated in Figure 2b. It may be seen that generation of Ru species in the polymer solution prior to intercalation affects the manner of polymer insertion and causes differentiation of the observed basal spacings. In particular, in the case of samples with lower PDDA loadings, intercalation appears to be hindered, as indicated by $\mathrm{d}_{001}$ values lower than in the corresponding Ru-free organomontmorillonites (Figure 2a, Table 1). In the case of samples $\mathrm{Mt} /(\mathrm{RU}+$ PDDA_0.05) and $\mathrm{Mt} /(\mathrm{Ru}+$ PDDA_0.25) the products of intercalation show structural inhomogeneity. In the first case a shoulder at higher $2 \Theta$ values indicates that there are polymer-poor regions in the final composite, in the second, next to the peak corresponding to $d_{001}$ value of $1.50 \mathrm{~nm}$, a strong reflection associated with $\mathrm{d}_{001}=1.99 \mathrm{~nm}$ shows that in a substantial part of Mt interlayers the intercalated polymer forms a double layer. In order to explain inhibition of PDDA intercalation in Ru catalysts with low PDDA content it should be recalled that ruthenium is generated within PDDA solution by treatment with large excess of $\mathrm{NaBH}_{4}$, which introduces into the solution a load of sodium cations, corresponding to ca. $5 \times \mathrm{CEC}$ of montmorillonite. Thus, competition with $\mathrm{Na}^{+}$prevents efficient intercalation of PDDA cations between the clay layers, the more, the lower is the polycation concentration. At higher PDDA contents $(x \geq 0.05)$, the well-known affinity of cationic polymer to clay matrix ensures that intercalation does occur, despite the fact that sodium concentration in the solution still exceeds that of PDDA polycations. Remarkably, in the sample with the highest nominal PDDA content, a substantial part of polymer assumes a bilayer packing $\left(\mathrm{d}_{001}=1.99 \mathrm{~nm}\right)$ much more pronounced than in the Ru-free sample.

The effect of Ru insertion by method II and III on XRD pattern of organomontmorillonite was studied for two levels of PDDA loadings, $x=0.05$, and $x=0.25$, and is shown in Figure $4 \mathrm{a}, \mathrm{b}$, respectively. In the former case, the XRD diagrams of Ru-containing composites do not differ in any significant manner from that of Mt/PDDA_0.05 sample, suggesting a similar manner of polymer packing in all samples. For $x=0.25$, addition of ruthenium by method II causes, similarly as in the case of method I, evolution, next to the peak corresponding to $d_{001}=1.56 \mathrm{~nm}$, of a reflection associated with $d_{001}=1.97 \mathrm{~nm}$, indicative of clay intercalated by a double layer of polymer. Thus, for the XRD-detectable structural order it is irrelevant whether Ru added to the polymer solution is reduced prior to (method I) or after (method II) intercalation. Application of method III to Ru insertion does not affect the XRD pattern of the parent Mt/PDDA samples in any meaningful way. 


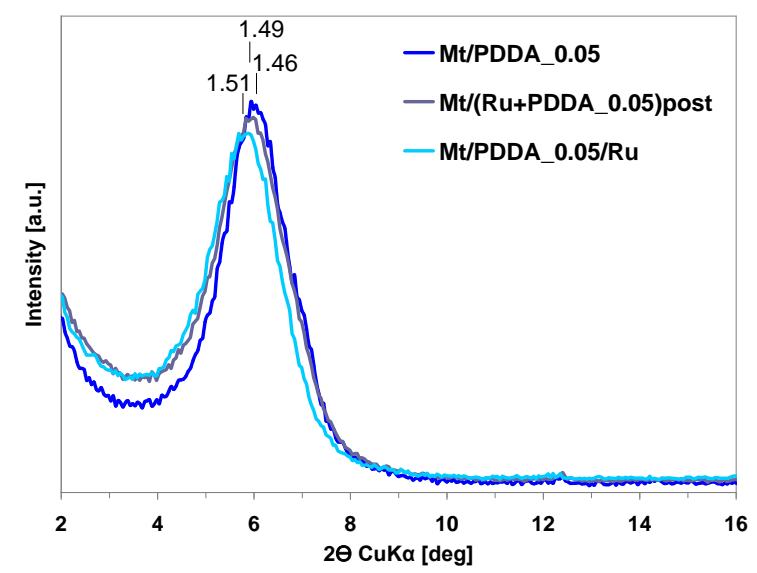

(a)

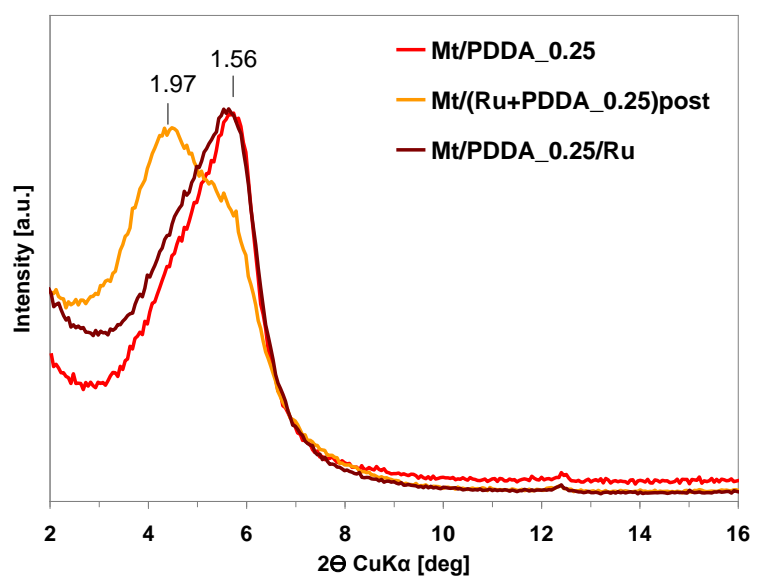

(b)

Figure 4. (a) Effect of Ru insertion by method II and method III on XRD pattern of Mt intercalated with PDDA cations ( $x=0.05)$; (b) effect of Ru insertion by method II and method III on XRD pattern of Mt intercalated with PDDA cations $(x=0.25)$.

In none of the Ru-containing samples can any reflections of metallic Ru be observed, which suggests that either the absolute content of $\mathrm{Ru}$ is too low to be detected by XRD, and/or that nanocrystalline character of $\mathrm{Ru}$ particles results in broadening of the diffraction peaks beyond possible detection.

TEM analysis of samples prepared by method I (Figure 5a-d) reveals that particles observed by AFM are, in fact, composed of much finer crystallites, of sizes in the range $2-4 \mathrm{~nm}$, the larger ones being preferentially formed in samples with low relative polymer content, the finer in materials rich in polymer component (Figure $5 \mathrm{a}-\mathrm{c}$ ). The effect is understandable in terms of the stronger dilution of $\mathrm{RuCl}_{3}$ precursor in the larger volumes of aqueous solution of PDDA. Moreover, in accordance with AFM data, upon decrease of the polymer content the Ru crystallites tend to form larger agglomerates. Figure 5d shows a HRTEM image of Ru crystallite with noticeable lattice fringes, representative for particles found in $\mathrm{Mt} /(\mathrm{Ru}+$ PDDA_0.05) sample. The interplanar distance of $0.205 \mathrm{~nm}$, derived from the fast Fourier transform (FFT), corresponds to (10-11) plane of hexagonal Ru lattice (ref. code 04-007-8640). Comparison of Figure 5e,g, which show Ru particles in samples Mt/(Ru + PDDA_0.05)post and Mt/PDDA_0.05/Ru, with Figure 5b, showing the Mt/(Ru + PDDA_0.05) material (all samples with the same nominal weight ratio of all components) enables assessment of the influence of various methods of Ru insertion on the form of generated ruthenium species. Thus, for PDDA/clay ratio of 0.05 , the preparation of the catalysts by mixing Ru source with the polymer solution followed by reduction prior to intercalation (method I) yields relatively largest $\mathrm{Ru}$ particles (dominating size ca. $3 \mathrm{~nm}$ ), the procedure with post-synthesis reduction of clay intercalated with polymer containing Ru precursor (method II) produces somewhat smaller crystallites (dominating size 2-2.5 nm), while adsorption of Ru on previously intercalated montmorillonite (method III) results in smallest, most dispersed species (dominating size 1-1.5 nm). Moreover, in sample prepared by method II, which enables co-intercalation of $\mathrm{Ru}^{3+}$ into the interlayer space, formation of very small $\mathrm{Ru}$ particles $(<1 \mathrm{~nm})$ in the interlayer space of clay is also observed (Figure $5 \mathrm{f})$. In the case of the maximum PDDA/clay weight ratio of 0.25 , comparison of appropriate TEM images shows that all methods of Ru incorporation result in the formation of very small nanoparticles, of ca. 1-2 nm (Figure 5c,h,i). 


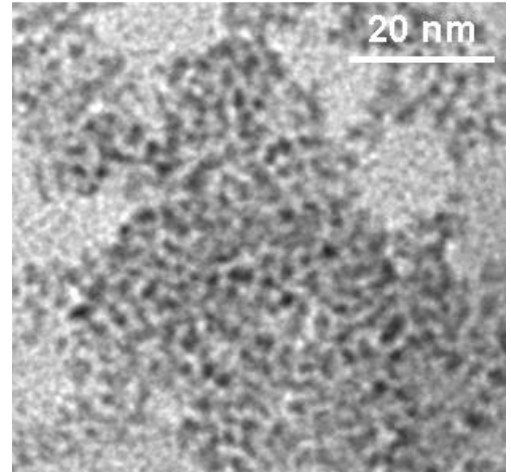

(a)

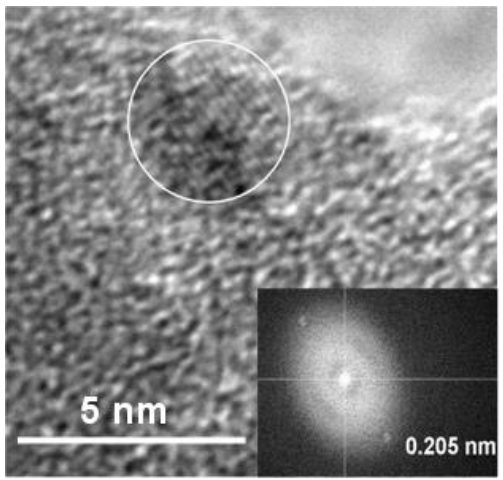

(d)

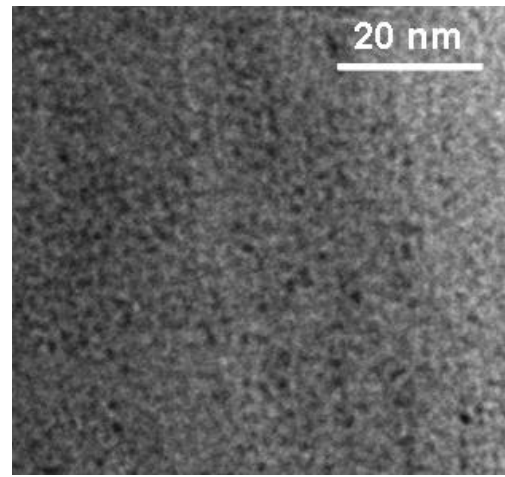

$(\mathrm{g})$

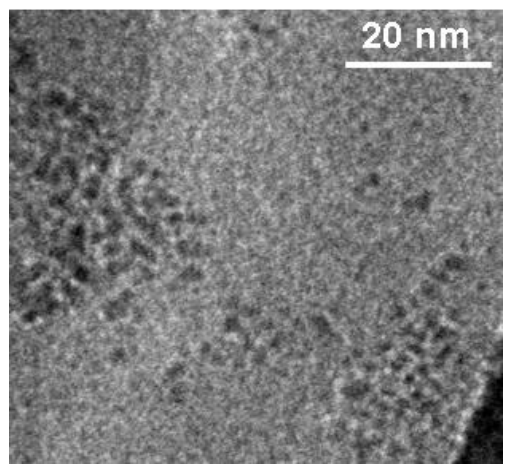

(b)

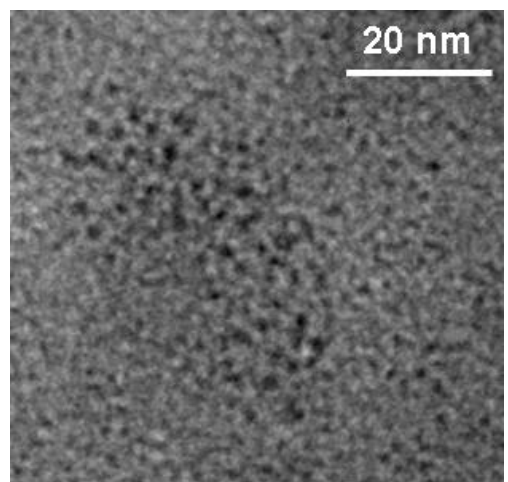

(e)

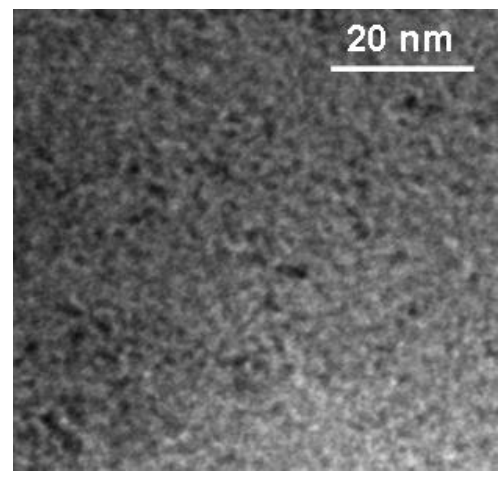

(h)

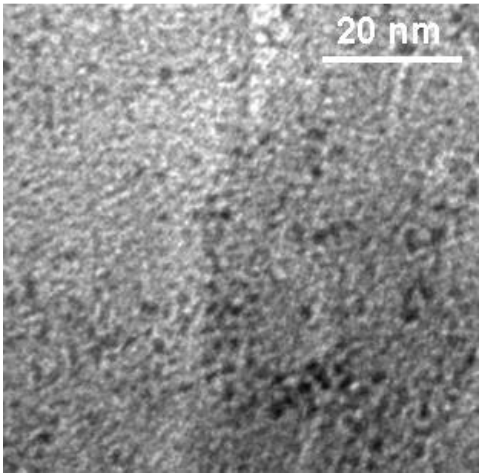

(c)

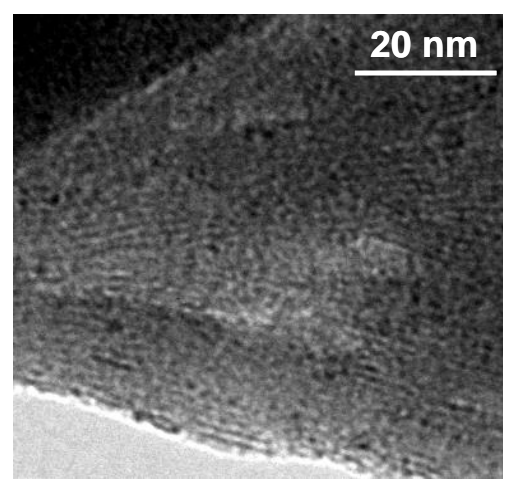

(f)

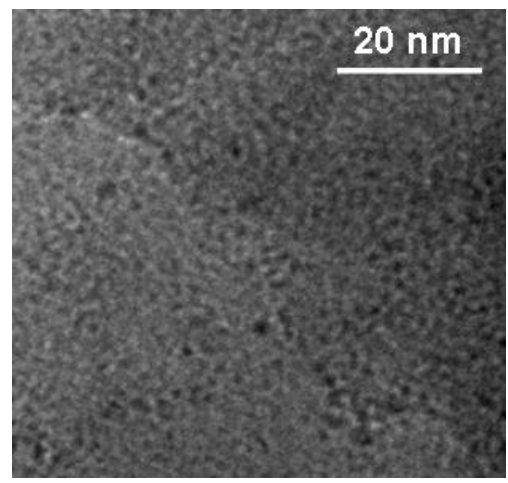

(i)

Figure 5. TEM images of $\mathrm{Ru}$ particles in: (a) $\mathrm{Mt} /(\mathrm{Ru}+$ PDDA_0.01); (b) $\mathrm{Mt} /(\mathrm{Ru}+$ PDDA_0.05); (c) $\mathrm{Mt} /(\mathrm{Ru}+$ PDDA_0.25); (d) HRTEM image of Ru crystallite in Mt/(Ru + PDDA_0.05) with corresponding FTT; (e,f) Mt/(Ru + PDDA_0.05)post; (g) Mt/PDDA_0.05/Ru; (h) Mt/(Ru + PDDA_0.25)post; (i) Mt/PDDA_0.25/Ru.

Investigated samples have been subjected to thermal analysis, in order to get insight into the amount of intercalated polymer (Figures 6 and 7, Table 1). Assignment of the TG/DSC effects follows the established views on the thermal properties of montmorillonite and its organic derivatives [28-30]. 


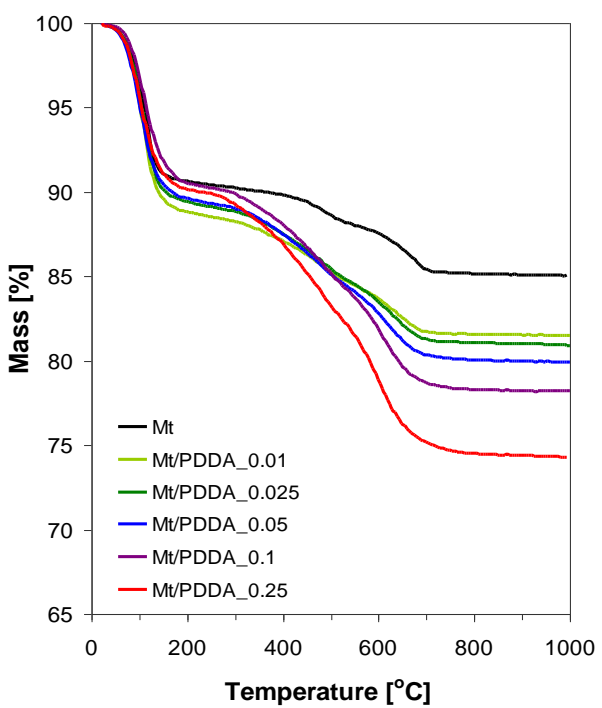

(a)

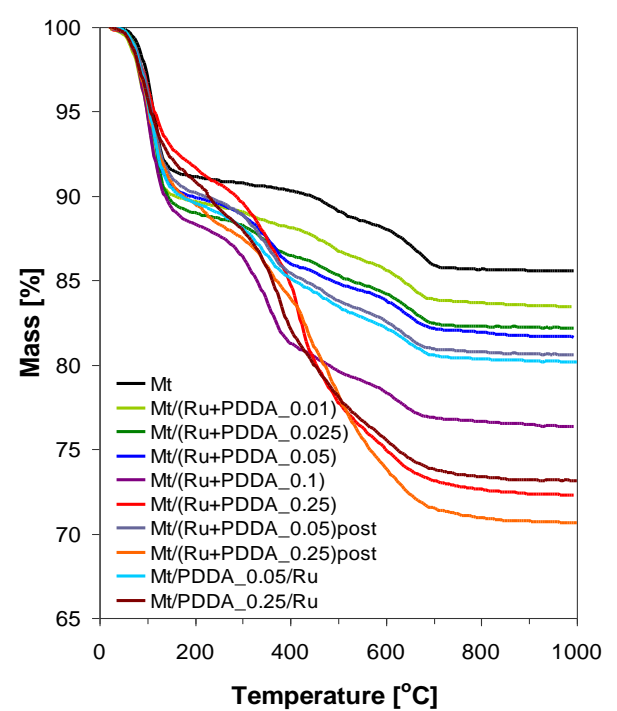

(b)

Figure 6. TG profiles of: (a) PDDA-intercalated montmorillonites; (b) Ru-loaded PDDA-intercalated montmorillonites. TG of parent montmorilllonite is provided for comparison.

The weight loss in the TG curves of PDDA-montmorillonite complexes occurs in two major temperature ranges, below and above ca. $200{ }^{\circ} \mathrm{C}$ (Figure 6a). In the parent montmorillonite the weight loss below $200{ }^{\circ} \mathrm{C}$ is associated with the departure of interlayer water coordinating exchangeable $\mathrm{Na}^{+}$ions, with a minor contribution from physisorbed water held in interparticle pores. The weight loss in the range $200-700{ }^{\circ} \mathrm{C}$ is due to dehydroxylation of montmorillonite layers reflected in the departure of water. It should be noted that the phenomena responsible for both steps overlap to some extent and there is no clear-cut border between them [31]. Thermal degradation/combustion of the organic component in PDDA-containing samples occurs above $200{ }^{\circ} \mathrm{C}$ and overlaps with the dehydroxylation of clay mineral layers. Table 1 provides the values of the observed weight losses in the temperature range $200-1000{ }^{\circ} \mathrm{C}$ for all samples whose TG profiles are presented in Figure $6 \mathrm{a}, \mathrm{b}$. The theoretical weight losses, estimated under the assumption that all introduced polymer has been incorporated into the sample, are given in brackets. For all samples, except of those with the highest employed PDDA/Mt ratio of 0.25 , the experimental values remain within the $\pm 15 \%$ margin from the theoretical ones, which, bearing in mind that the end of the clay dehydration step may extend above the arbitrary limit of $200{ }^{\circ} \mathrm{C}$, indicates that practically all PDDA enters the montmorillonite structure (Table 1). This is the case even for the Mt/PDDA_0.01 sample, with the lowest PDDA loading, corresponding to ca. $7 \%$ of the clay CEC, and demonstrates the high affinity of the polycation towards the clay matrix. In the case of samples with PDDA/Mt ratio of 0.25 , the amount of added PDDA corresponds to ca. $180 \%$ of CEC, therefore Table 1 provides also information of the theoretical mass loss expected if the PDDA content in the composite is less than the added total and corresponds to the maximum CEC-allowable value. It may be seen that for Mt/PDDA_0.25 and Mt/PDDA_0.25/Ru samples, in which Ru-free PDDA solution was used for intercalation, the observed weight loss indicates that the samples retain less than the introduced amount of PDDA, close to the CEC dependent value, the excess being apparently removed upon washing. In contrast, in Mt/(Ru + PDDA_0.25) and Mt/(Ru + PDDA_0.25)post samples, in which intercalated PDDA solution contained either Ru particles or $\mathrm{RuCl}_{3}$, the amount of incorporated PDDA is higher, suggesting that in the presence of Ru species more PDDA can be loaded into the clay matrix. In both materials the stage of final washing of the sample comes after the formation of Ru nanoparticles. Possibly, the polymer interacting with $\mathrm{Ru}$ nanoparticles is more rigid and less prone to removal upon washing than that present in the Ru-free 
system. The presence of larger amount of polymer also explains the appearance of a bilayer PDDA packing in these samples, evidenced by XRD (Figures $2 b$ and $4 b$ ).

Valuable information as to the nature of the interaction between the components of the composites, depending on the method of Ru insertion, can be derived from the analysis of DSC profiles (Figure 7). In parent Mt (Figure 7a), the endotherm peaking near $110{ }^{\circ} \mathrm{C}$ accompanies the release of interlayer water associated with the compensating cations, the endotherm at $678^{\circ} \mathrm{C}$ is due to the dehydroxylation of the montmorillonite layers, and a small S-shaped endo-exotherm centered around $900{ }^{\circ} \mathrm{C}$ indicates structural rearrangements accompanying the formation of new phases. With addition of PDDA component, new maxima of exothermic character appear, at 360,423 , and $588^{\circ} \mathrm{C}$ (Figure 7a). As expected, their intensity increases with the amount of added polymer. The first two are attributed to the decomposition/combustion of PDDA, the third is attributed to the combustion of the carbonaceous deposit formed during incomplete oxidation of the organic matter at the previous stage of polymer destruction.

The effect of various manners of Ru addition to samples with the PDDA/clay ratio of 0.05 is shown in Figure $7 \mathrm{~b}$. The overall appearance of the DSC profiles for Ru-containing samples is similar, irrespective of the preparation method. In all cases it is obvious that in the presence of $\mathrm{Ru}$, the combustion of the PDDA component is shifted to lower temperature, as manifested by the strong exotherm around $350{ }^{\circ} \mathrm{C}$, accompanied by the disappearance/suppression of 423 and $588 \mathrm{~cm}^{-1}$ maxima in Ru-free matrix. This effect may be attributed to the catalytic action of $\mathrm{Ru}$ nanoparticles, known to facilitate total oxidation of organic matter [32]. Moreover, despite the general similarity of DSC curves for Ru-containing samples with PDDA/clay ratio of 0.05 prepared by different methods, there is a difference between the positions of maxima relevant to PDDA combustion. The ease of PDDA component combustion follows the order: Mt/PDDA_0.05/Ru $\left(344^{\circ} \mathrm{C}\right)>\mathrm{Mt} /(\mathrm{Ru}+$ PDDA_0.05)post $\left(354^{\circ} \mathrm{C}\right)>\mathrm{Mt} /\left(\mathrm{Ru}+\right.$ PDDA_0.05) $\left(369^{\circ} \mathrm{C}\right)$. In view of the TEM data it appears that the smaller the Ru nanoparticles the higher is their efficiency in PDDA component combustion. The effect is understandable in terms of the growing Ru-PDDA interface upon diminution of Ru species.

Thermal properties of samples with the nominal PDDA/clay ratio of 0.25 (Figure 7c) show considerably larger differences. Although the general effect of accelerated combustion of the organic matter, manifested by the downward shift of associated exothermic maxima, is still present, it is not so strong and depends on the manner of Ru insertion. Only in the case of Mt/PDDA_0.25/Ru sample, prepared by method III, and containing considerably less PDDA than the nominal loading, the DSC profile resembles that of the Mt/PDDA_0.05/Ru counterpart. Also here a single exothermic maximum is observed at $384{ }^{\circ} \mathrm{C}$, indicating that efficient combustion, leaving no significant char, occurs below $400{ }^{\circ} \mathrm{C}$. A different situation is found with samples Mt/(Ru + PDDA_0.25) and Mt/(Ru + PDDA_0.25)post, prepared by method I and II. Here, the downward shift is practically limited to the effect related to the combustion of carbonaceous deposit, while the first maximum appears at a similar temperature around $420^{\circ} \mathrm{C}$. The observed phenomena suggest that in the composites most heavily loaded with PDDA, in which the polymer areas in contact with Ru species lose on importance with respect to PDDA fraction not affected by the Ru presence, it is the diffusion of oxygen required for the combustion, that controls the evolution of DSC profiles. Thus, the first DSC effect around $420^{\circ} \mathrm{C}$ appears at a similar position as in the Ru-free material, because penetration of polymer-loaded material by oxygen, to reach the Ru-containing areas, is hindered by the large volume of polymer chains. On the other hand, at higher temperature, when most of the PDDA has been burned off and the remnants turned into the carbonaceous deposit, new diffusion paths for air become available, and the effect of $\mathrm{Ru}$ species may become visible in the form of lowering of the char combustion temperature, more pronounced in the material with the relatively smaller initial content of polymer, i.e., $\mathrm{Mt} /(\mathrm{Ru}+$ PDDA_0.25)post. 


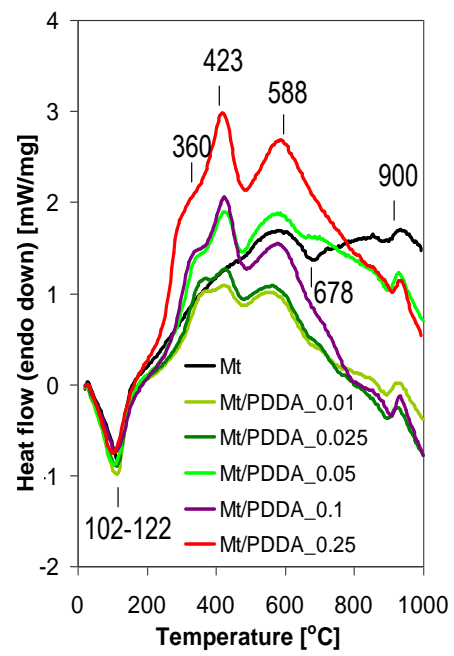

(a)

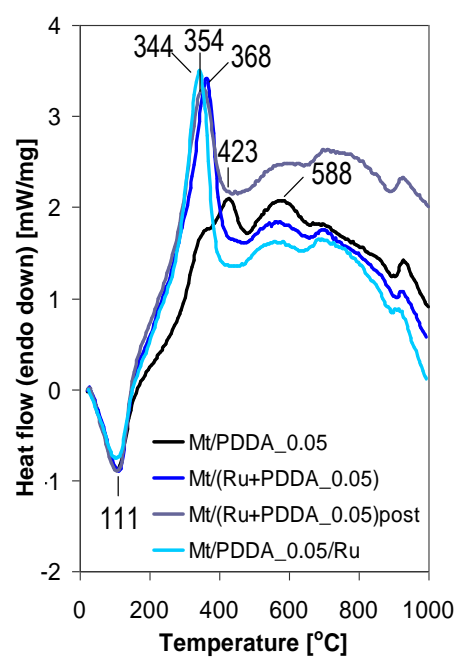

(b)

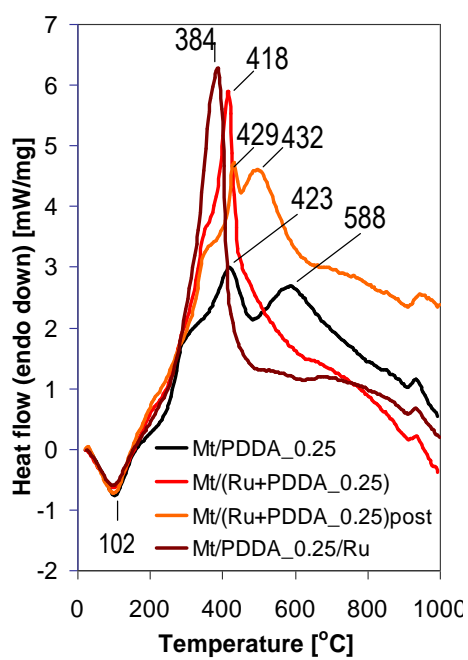

(c)

Figure 7. DSC profiles of: (a) PDDA-intercalated montmorillonites; (b) composites with PDDA/clay weight ratio of 0.05 ; (c) composites with PDDA/clay weight ratio of 0.25 .

\subsection{Catalytic Testing}

Among many organic transformations, catalytic hydrogenation of $\mathrm{C}=\mathrm{O}$ bond is one of the most important synthetic methods enabling the preparation of a great number of useful products [33]. Hydrogenation of 2-butanone yields 2-butanol (Figure 8), a commonly used industrial solvent.<smiles>CCC(C)=O</smiles><smiles>CCC(C)O</smiles>

Figure 8. Scheme of 2-butanone hydrogenation.

The results of the catalytic tests of 2-butanone hydrogenation carried out in this work are summarized in Figure 9. Figure 9a shows the dependence of 2-butanone conversion for the series of catalysts obtained by method I, differing in the polymer/clay weight ratio. No conversion of 2-butanone was observed on parent Mt or an organoclay not containing ruthenium. It is apparent that the highest activity (100\% conversion) is obtained over the Mt/(Ru + PDDA_0.05) catalyst with an intermediate polymer content. It has been repeatedly demonstrated by theoretical and experimental studies that maximum catalytic activity in various processes catalyzed by Ru nanoparticles can be achieved by optimization of the particle size, so as to obtain the highest population of the active sites favorable for the catalytic transformation [34-36]. In the present work, the physicochemical characterization revealed that Ru particle sizes in this series vary in the 2-4 nm range, showing a tendency to become smaller upon increase of the relative polymer content. The maximum activity observed for the catalyst with intermediate PDDA loading, hence intermediate Ru particle dimensions, may be taken as an indication that also in the present study the size of Ru species plays an important role. However, it appears that it is not the only factor influencing the catalytic activity. Figure 9a shows that a particularly rapid fall of activity occurs in materials heavily loaded with PDDA, i.e., $\mathrm{Mt} /(\mathrm{Ru}+$ PDDA_0.1) and Mt/(Ru + PDDA_0.25). Although in these samples Ru crystallites become smaller, so the fall of activity is in line with the hypothesis of the existence of an optimum Ru crystal size, but it should be remembered that the large volume of polymer present in these materials is likely to hinder the access of reagents to Ru particles. Therefore, the diffusional limitations within the catalyst structure are another probable cause of the observed strong decrease of catalytic activity. 


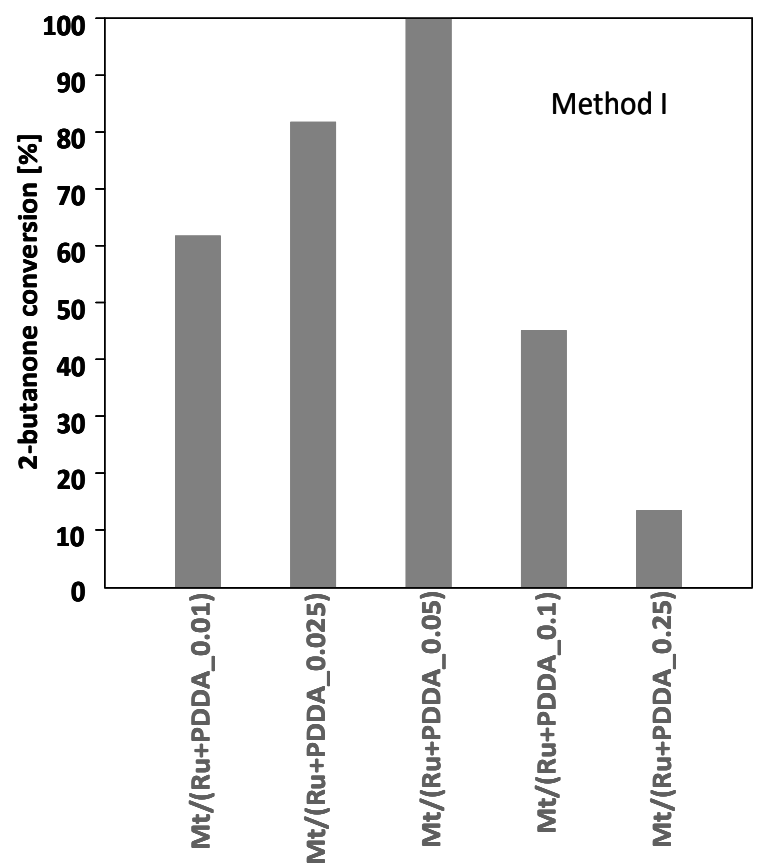

(a)

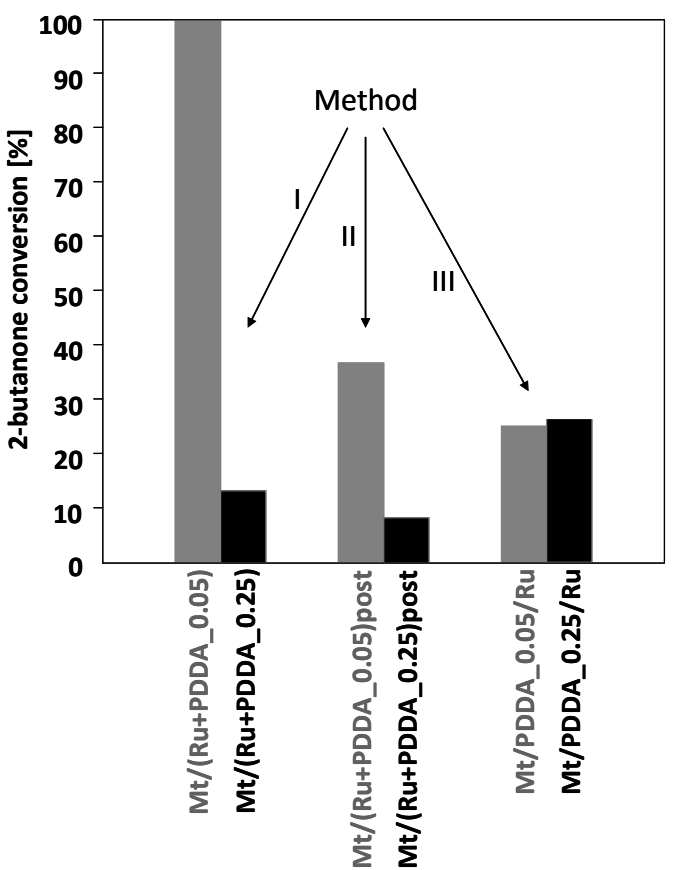

(b)

Figure 9. (a) Catalytic activity in 2-butanone hydrogenation of $\mathrm{Mt} /(\mathrm{Ru}+$ PDDA_ $x)$ composites; (b) comparison of catalytic activity of composites prepared by different methods, for polymer/clay weight ratio of 0.05 and 0.25 . In all cases selectivity to 2 -butanol is $100 \%$.

Comparison of catalytic experiments carried out for composites with the same polymer loading, but prepared with different methods of Ru insertion, sheds additional light on the role of particular factors in determining the catalytic performance of Ru-clay-polymer composites (Figure 9b). Thus, in the case of materials prepared with the PDDA/clay weight ratio of 0.05 , which exploits only about $35 \%$ of the clay CEC, the order of catalytic activity follows the order of Ru particles size, the most active catalyst being the one obtained by method I, with most particles having ca. $3 \mathrm{~nm}$, followed by samples obtained by methods II and III, with lower particle sizes. In samples with the highest investigated PDDA/clay ratio of 0.25 (loading in excess to CEC), which all contain small $\mathrm{Ru}$ particles $(\leq 2 \mathrm{~nm})$, catalytic performance depends strongly on the manner of catalysts preparation. Thus, composites prepared by methods I and II, i.e., $\mathrm{Mt} /(\mathrm{Ru}+\mathrm{PDDA}$-0.25) and Mt/(Ru + PDDA_0.25)post, perform significantly poorer than their counterparts with PDDA/clay ratio equal 0.05 . The effect is attributed to the high polymer content, making it difficult for the reagents to reach Ru particles. In contrast, in the case of sample prepared by method III, Mt/PDDA_0.25/Ru, the catalytic activity is comparable with that observed for the Mt/PDDA_0.05/Ru with much lower PDDA content. Moreover, the sample displays the highest activity among the materials with high PDDA loading. The latter may be due in part to the lower PDDA amount in this sample, causing lesser blocking, and in part to the manner of Ru addition. When Ru is introduced by adsorption onto the already formed Mt/PDDA composite, the Ru particles, although very small, and as such far from being optimally active, are likely to remain at the surface or in the near-to-surface area of the catalyst, thus eliminating diffusional limitations typical of PDDA-rich materials prepared by methods I and II. This explains both the higher activity of the Mt/PDDA_0.25/Ru catalyst in comparison with samples prepared by methods I and II, and the lack of difference in catalytic performance with respect to the Mt/PDDA_0.05/Ru catalyst.

Finally, we would like to underline, that the Ru catalysts based on PDDA/clay composites described in this work are active under exceptionally mild conditions. The vast majority of the reported studies on 2-butanone heterogeneous catalytic hydrogenation have been investigated at elevated hydrogen pressure 
and/or temperature, while the conditions used in the present work involved normal hydrogen pressure and room temperature [24,37-39]. Moreover, all catalysts obtained by method I, with PDDA/clay weight ratio $\leq 0.05$, outperform ruthenium catalysts of similar Ru content, supported on mesoporous silica of SBA-15 type and/or conventional high surface area silica, described in our recent work, which, in the same catalytic system, converted 51 and $37 \%$ 2-butanone, respectively [40].

\section{Conclusions}

The amount of PDDA retained by montmorillonite is governed by the CEC of clay. When the quantity of added PDDA is below CEC, practically all polycations become trapped by the clay matrix; when it is higher than $C E C$, most of the excess polymer can be removed at the stage of washing.

It is postulated that, due to the acidic $\mathrm{pH}$ of PDDACl solution, in samples with lower PDDA loading, next to the single layer of polycations, also hydronium ions are intercalated and contribute to the observed basal spacing.

Generation of Ru species in the polymer solution prior to intercalation (method I) hinders intercalation of polycations into composites with low PDDA/clay ratio, because of the competitive exchange with $\mathrm{Na}^{+}$from the $\mathrm{NaBH}_{4}$ solution used for $\mathrm{Ru}^{3+}$ reduction. No such effect is observed in composites obtained by procedures in which treatment with $\mathrm{NaBH}_{4}$ is carried out after polymer intercalation (methods II and III).

TEM study reveals that Ru crystallites are quite small, from less than 1 to $4 \mathrm{~nm}$, depending on the preparation method. In preparative routes involving mixing of Ru source with the polycation solution (methods I and II), the size of Ru particles diminishes upon increase of the relative PDDA content. The finest particles are formed upon adsorption of Ru onto clay intercalated with PDDA (method III).

The presence of Ru changes thermal properties of the polymer-clay composites, as it catalyzes the combustion of the organic component. The effect is more pronounced in materials with lower relative PDDA content and smaller Ru particles, i.e., under conditions providing higher polymer-Ru interface.

The catalysts are active in hydrogenation of 2-butanone under very mild conditions (room temperature, atmospheric pressure of $\mathrm{H}_{2}$, and aqueous solution). For best catalytic performance both the size of $\mathrm{Ru}$ particles and the load of polymer have to be optimized. Superior catalytic properties are obtained over the composite with intermediate crystal size and intermediate PDDA load, prepared by generation of metallic $\mathrm{Ru}$ species in the polymer solution prior to intercalation. This method offers an easy way of controlling the crystal size by modification of $\mathrm{Ru} / \mathrm{PDDA}$ ratio.

Results of this study underline the significance of fine details in the preparative procedures aiming at synthesis of ternary clay/polymer/metal nanoparticles composites. The presented findings provide insight into the factors enabling control of the materials properties and are expected to be of use in the design of related systems.

Author Contributions: M.Z. and H.P. synthesized the materials, M.Z., D.D., B.D.N., M.N.-R., G.M., L.L.-D., and H.P., performed the experiments, E.M.S. supervised the research and wrote the draft of the manuscript, all authors contributed to the final version of the paper.

Funding: Development of the catalytic set-up for liquid phase hydrogenation was funded by the NCN (Narodowe Centrum Nauki project UMO-2012/07/B/ST5/00770). M.Z. and H.P acknowledge mobility support within Polish-Slovak joint project 2016-2018 under the PAS-SAS agreement. H.P acknowledges the financial support of the Slovak Grant Agency (VEGA 2/0141/17).

Conflicts of Interest: The authors declare no conflicts of interest. The founding sponsors had no role in the design of the study; in the collection, analyses, or interpretation of data; in the writing of the manuscript, and in the decision to publish the results. 


\section{References}

1. McKenna, K.P. Unique Bonding in Nanoparticles and Powders. In Nanoscale Materials in Chemistry, 2nd ed.; Klabunde, K.J., Richards, R.M., Eds.; John Wiley \& Sons, Inc.: Hoboken, NJ, USA, 2009; pp. 15-36, ISBN 978-0-470-22270-6.

2. Li, Y.; Boone, E.; El-Sayed, M.A. Size Effects of PVP-Pd Nanoparticles on the Catalytic Suzuki Reactions in Aqueous Solution. Langmuir 2002, 18, 4921-4925. [CrossRef]

3. Rao, C.N.R.; Kulkarni, G.U.; Thomas, P.J.; Edwards, P.P. Size-Dependent Chemistry: Properties of Nanocrystals. Chem. Eur. J. 2002, 8, 28-35. [CrossRef]

4. Crooks, R.M.; Zhao, M.; Sun, L.; Chechik, V.; Lee, K.Y. Dendrimer-Encapsulated Metal Nanoparticles: Synthesis, Characterization, and Applications to Catalysis. Acc. Chem. Res. 2001, 34, 181-190. [CrossRef] [PubMed]

5. Bönnemann, H.; Richards, R.M. Nanoscopic Metal Particles—Synthetic Methods and Potential Applications. Eur. J. Inorg. Chem. 2001, 2455-2480. [CrossRef]

6. Ford, W.T. Inorganic-Organic Composites. In Nanoscale Materials in Chemistry, 2nd ed.; Klabunde, K.J., Richards, R.M., Eds.; John Wiley \& Sons, Inc.: Hoboken, NJ, USA, 2009; pp. 369-403, ISBN 978-0-470-22270-6.

7. Li, Y.; El-Sayed, M.A. The Effect of Stabilizers on the Catalytic Activity and Stability of Pd Colloidal Nanoparticles in the Suzuki Reactions in Aqueous Solution. J. Phys. Chem. B 2001, 105, 8938-8943. [CrossRef]

8. Králik, M.; Biffis, A.J. Catalysis by metal nanoparticles supported on functional organic polymers. Mol. Catal. A 2001, 177, 113-13810. [CrossRef]

9. Liu, P. Polymer modified clay minerals: A review. Appl. Clay Sci. 2007, 38, 64-76. [CrossRef]

10. Chiu, C.W.; Huang, T.K.; Wang, Y.C.; Alamani, B.G.; Lin, J.J. Intercalation strategies in clay/polymer hybrids. Prog. Polym. Sci. 2014, 39, 443-485. [CrossRef]

11. Zhou, C.H. An overview on strategies towards clay-based designer catalysts for green and sustainable catalysis. Appl. Clay Sci. 2011, 53, 87-96. [CrossRef]

12. Azeez, A.A.; Rhee, K.Y.; Park, S.J.; Hui, D. Epoxy clay nanocomposites - processing, properties and applications: A review. Compos Part B 2013, 45, 308-320. [CrossRef]

13. Napruszewska, B.D.; Michalik-Zym, A.; Dula, R.; Bielanska, E.; Rojek, W.; Machej, T.; Socha, R.P.; Lityńska-Dobrzyńska, L.; Bahranowski, K.; Serwicka, E.M. Composites derived from exfoliated Laponite and $\mathrm{Mn}-\mathrm{Al}$ hydrotalcite prepared in inverse microemulsion: A new strategy for design of robust VOCs combustion catalysts. Appl. Catal. B 2017, 211, 46-56. [CrossRef]

14. Napruszewska, B.D.; Michalik-Zym, A.; Rogowska, M.; Bielańska, E.; Rojek, W.; Gaweł, A.; Wójcik-Bania, M.; Bahranowski, K.; Serwicka, E.M. Novel Montmorillonite/ $\mathrm{TiO}_{2} / \mathrm{MnAl}-$ Mixed Oxide Composites Prepared from Inverse Microemulsions as Combustion Catalysts. Materials 2017, 10, 1326. [CrossRef] [PubMed]

15. Papp, S.; Szücs, A.; Dékány, I. Preparation of $\mathrm{Pd}^{0}$ nanoparticles stabilized by polymers and layered silicate. Appl. Clay Sci. 2001, 19, 155-172. [CrossRef]

16. Papp, S.; Dékány, I.; Szél, J.; Oszkó, A. Synthesis of Polymer-Stabilized Nanosized Rhodium Particles in the Interlayer Space of Layered Silicates. Chem. Mater. 2004, 16, 1674-1685. [CrossRef]

17. Zhao, X.; Mai, Z.; Kang, X.; Zou, X. Direct electrochemistry and electrocatalysis of horseradish peroxidase based on clay-chitosan-gold nanoparticle nanocomposite. Biosens. Bioelectron. 2008, 23, 1032-1038. [CrossRef] [PubMed]

18. Jlassi, K.; Singh, A.; Aswal, D.K.; Losno, R.; Benna-Zayani, M.; Chehimi, M.M. Novel, ternary clay/polypyrrole/silver hybrid materials through in situ photopolymerization. Colloids Surf. A 2013, 439, 193-199. [CrossRef]

19. Xu, M.; Zhao, J.; Shu, G.; Liu, Q.; Zeng, M. Heterogeneous Catalytic Composites from Palladium Nanoparticles in Montmorillonite Intercalated with Poly (Vinyl Pyrrolidone) Chains. Polymers 2018, 10, 669. [CrossRef]

20. Fan, M.; Wang, R.; Jia, S. Controllable synthesis of iron nanoparticles on polyethylenimine-modified montmorillonite: Dependence on the amine protonation extent. Appl. Clay Sci. 2018, 162, 418-427. [CrossRef]

21. Brigatti, M.F.; Galán, E.; Theng, B.K.G. Structure and Mineralogy of Clay Minerals. In Handbook of Clay Science, 2nd ed.; Bergaya, F., Lagaly, G., Eds.; Elsevier: Amsterdam, The Netherlands, 2013; Part A, pp. 21-81, ISBN 978-0-08-098259-5. 
22. Dautzenberg, H.; Gornitz, E.; Jaeger, W. Synthesis and characterization of poly (diallyldimethylammonium chloride) in a broad range of molecular weight. Macromol. Chem. Phys. 1998, 199, 1561-1571. [CrossRef]

23. Michel, C.; Gallezot, P. Why Is Ruthenium an Efficient Catalyst for the Aqueous-Phase Hydrogenation of Biosourced Carbonyl Compounds? ACS Catal. 2015, 5, 4130-4132. [CrossRef]

24. Breen, J.P.; Burch, R.; Griffin, K.; Hardacre, C.; Hayes, M.; Huang, X.; O’Brien, S.D. Bimetallic effects in the liquid-phase hydrogenation of 2-butanone. J. Catal. 2005, 236, 270-281. [CrossRef]

25. Breen, C.; Rawson, J.O.; Mann, B.E. Adsorption of polycations on clays: An in situ study using ${ }^{133} \mathrm{Cs}$ solution-phase NMR. J. Mater. Chem. 1996, 6, 253-260. [CrossRef]

26. Churchman, G.J. Formation of complexes between bentonite and different cationic polyelectrolytes and their use as sorbents for non-ionic and anionic pollutants. Appl. Clay Sci. 2002, 21, 177-189. [CrossRef]

27. Czímerová, A.; Jankovič, L.; Madejová, J.; Čeklovský, A. Unique Photoactive Nanocomposites Based on Rhodamine 6G/Polymer/Montmorillonite Hybrid Systems. J. Polym. Sci. B Polym. Phys. 2013, 51, 1672-1679. [CrossRef]

28. Meunier, A. Clays; Springer-Verlag: Berlin, Heidelberg, Germany, 2005; pp. 196-198, ISBN 978-3-540-21667-4.

29. Langier-Kuźniarowa, A. Thermal Analysis of Organoclay Complexes. In Organo Clay Complexes and Interactions; Yariv, S., Cross, H., Eds.; Marcel Dekker: New York, NY, USA, 2001; pp. 273-344, ISBN 0-8247-0586-6.

30. Yariv, S. The role of charcoal on DTA curves of organo-clay complexes: an overview. Appl. Clay Sci. 2004, 24, 225-236. [CrossRef]

31. Fajnor, V.Š.; Jesenák, K. Differential thermal analysis of montmorillonite. J. Therm. Anal. 1996, 46, 489-493. [CrossRef]

32. Kamiuchi, N.; Mitsui, T.; Muroyama, H.; Matsui, T.; Kikuchi, R.; Eguchi, K. Catalytic combustion of ethyl acetate and nano-structural changes of ruthenium catalysts supported on tin oxide. Appl. Catal. B 2010, 97, 120-126. [CrossRef]

33. Rylander, P.N. Catalytic Hydrogenation in Organic Syntheses; Academic Press: New York, NY, USA, 1979; pp. 82-112, ISBN 978-0-12-605355-5.

34. Gavnholt, J.; Schiøtz, J. Structure and reactivity of ruthenium nanoparticles. Phys. Rev. B 2008, 77, 035404-1-035404-10. [CrossRef]

35. Karim, A.M.; Prasad, V.; Mpourmpakis, G.; Lonergan, W.W.; Frenkel, A.I.; Chen, J.G.; Vlachos, D.G. Correlating Particle Size and Shape of Supported $\mathrm{Ru} / \gamma-\mathrm{Al}_{2} \mathrm{O}_{3}$ Catalysts with $\mathrm{NH}_{3}$ Decomposition Activity. J. Am. Chem. Soc. 2009, 131, 12230-12239. [CrossRef] [PubMed]

36. Bu, J.; Liu, J.L.; Chen, X.Y.; Zhuang, J.H.; Yan, S.R.; Qiao, M.H.; He, H.Y.; Fan, K.N. Ru/SBA-15 catalysts for partial hydrogenation of benzene to cyclohexene: Tuning the Ru crystallite size by Ba. Catal. Commun. 2008, 9, 2612-2615. [CrossRef]

37. Akpa, B.S.; D'Agostino, C.; Gladden, F.L.; Hindle, K.; Manyar, H.; McGregor, J.; Li, R.; Neurock, M.; Sinha, N.; Sitt, E.H; et al. Solvent effects in the hydrogenation of 2-butanone. J. Catal. 2012, 289, 30-41. [CrossRef]

38. Wan, H.; Vitter, A.; Chaudhari, R.V.; Subramaniam, B. Kinetic investigations of unusual solvent effects during $\mathrm{Ru} / \mathrm{C}$ catalyzed hydrogenation of model oxygenates. J. Catal. 2014, 309, 174-184. [CrossRef]

39. Jones, D.R.; Iqbal, S.; Kondrat, S.A.; Lari, G.M.; Miedziak, P.J.; Morgan, D.J.; Parker, S.F.; Hutchings, G.J. An investigation of the effect of carbon support on ruthenium/carbon catalysts for lactic acid and butanone hydrogenation. Phys. Chem. Chem. Phys. 2016, 18, 17259-17264. [CrossRef] [PubMed]

40. Duraczyńska, D.; Michalik-Zym, A.; Napruszewska, B.D.; Dula, R.; Socha, R.P.; Lityńska-Dobrzyńska, L.; Gaweł, A.; Bahranowski, K.; Serwicka, E.M. Efficient and Versatile Ru/SBA-15 Catalysts for Liquid-hase Hydrogenation of the $\mathrm{C}=\mathrm{C}$ and $\mathrm{C}=\mathrm{O}$ Bonds under Mild Conditions. ChemistrySelect 2016, 1, 2148-2155. [CrossRef]

(C) 2018 by the authors. Licensee MDPI, Basel, Switzerland. This article is an open access article distributed under the terms and conditions of the Creative Commons Attribution (CC BY) license (http:/ / creativecommons.org/licenses/by/4.0/). 